\title{
COMMON DATA ADMINISTRATION, DATA MANAGEMENT, AND DATA ALIGNMENT AS A NECESSARY REQUIREMENT FOR COUPLING C4ISR SYSTEMS AND M\&S SYSTEMS
}

\author{
Andreas TOLK
}

\section{Simulation Applications and C4ISR Systems}

There is a strong necessity to solve the interoperability issue between information systems used for Command, Control, Computing, Communications, Intelligence, Surveillance, and Intelligence (C4ISR) and combat simulation systems. Interoperability is an operationally driven requirement in several application domains of combat simulation systems and it is stated in milestone documents such as the US DoD Modeling and Simulation Master Plan ${ }^{1}$ and the NATO Modeling and Simulation Master Plan. ${ }^{2}$

- Within the simulation application domain of training and exercises appropriate simulation systems are used to create a synthetic environment for the trainees that is supplied to the C4ISR systems used by the soldiers in the field to "train them as they fight." Hence, the simulation systems used for computer-assisted exercises (CAX) have to be able to provide the necessary input to the C4ISR systems used by the trainees. Additionally, the orders given by the trainees to the simulated units have to be brought into the simulation systems as efficiently as possible, which implies that, as a minimum, the orders have to be transferable from the C4ISR application to the embedded simulated environment.

- Modeling and simulation (M\&S) have also been applied successfully in simulation-based acquisition (SBA) for several years. Simulation systems are used to build a synthetic environment that dynamically generates test data for the C4ISR systems in the acquisition process. Furthermore, M\&S is used to simulate new components to be introduced into the C4ISR systems. This application also leads to the requirement for interoperability between the 
simulation and the embedded system to be tested.

- While the application domains mentioned above can be considered already well established, a relatively new domain is "bringing military operations research (MOR) methods back to war:" the simulation application domain of online support to real military operations. This new application domain uses methods of M\&S to support the military decision-making and command and control processes. To achieve this, methods of $M \& S$ are integrated into the operational C4ISR systems to support the analysis of alternative courses of action, to check the consistency of operational plans, to control the development of operations, etc.

\section{State-of-the-Art Solutions}

However, the challenges that a system designer faces are still big. As a matter of fact, every integration effort establishes a new project with new or reinvented solutions, own - and often proprietary - methods and tools, etc. Surprisingly, to the decision maker these interoperability issues between C4ISR Systems and Simulations have the appearance of being pure technical problems, and thus are relegated to the backwaters of M\&S policy. While the focus is on the reuse of components, we are still on the level of "home workers" that prepare them for the intended reuse. Although, under the aegis of the Simulation Interoperability Standardization Organization (SISO) a study group has dealt with the issues of interoperability between C4I and simulation systems, ${ }^{3}$ such interface building efforts are still performed on a more or less ad hoc proprietary basis and rarely any real guidance exists, which leads to double work, reinvention of solutions, and last but not least rigid bridges between the systems. And instead of "system of systems" that we aim to build, nowadays we often face a "bunch of systems." This is especially true for C4ISR system to M\&S system interface solutions.

On the other hand, integration has always been an issue where different legacy or in parallel developed information technology based solutions are brought together. The commercial, as well as, the academic worlds have arrived at some interesting solutions that are applicable to military problems. And, as the problem of integrating C4ISR systems and M\&S systems during the actual first phase of the integration process is a problem of establishing information exchange over the interfaces developed between the respective systems, the ideas of federated databases can be successfully applied. The theory of federated databases deals with the challenge of merging distributed, heterogeneous, and autonomous data sources in such a way that they can be used by other applications. To this end, a rigorous common management process accompanies the technical solution. For a general introduction to this domain, a reference to the work of Sheth and Larson is recommended. ${ }^{4}$ To author's 
knowledge, the most comprehensive introduction and overview is Stefan Conrad's book on federated database systems, which is written in German. ${ }^{5}$ The applicability of respective solutions to military problems is described by the author of this paper in the Simulation Interoperability Workshop Paper on "Bridging the Data Gap" between C4ISR systems and M\&S systems. ${ }^{6}$

\section{A Necessity for Common Data Engineering}

A common problem to all the different solutions is that the system designer responsible for the integration has to know what data is located where, the meaning of data and its context, and what format the data has to be transformed to so that it can be used in the respective distributed applications within the overall system. Generating answers to these questions is the objective of data administration, data management, data alignment, and data transformation, which can be defined as the building blocks of a new role in the interoperability process: the task of data engineering. The first three of these tasks can be standardized and used in a general manner. Only the task of data transformation is system dependent, but even for this task it will be shown that a general solution exists.

As already mentioned, these are the necessary first steps in a broader interoperability framework. Although this paper focuses mainly on the data issue of interoperability, a more general framework and the future work perceived by the author will be described as well.

In the context of this paper, the author defines the respective terms as follows:

- Data Administration is the process of managing the information exchange needs that exist within a group of systems, including the documentation of the source, format, context of validity, fidelity, and credibility of the data. Data Administration, therefore, is part of the overall information management process.

- Data Management is planning, organizing and managing of data by defining and using rules, methods, tools and respective resources to identify, clarify, define and standardize the meaning of data in terms of relations.

- Data Alignment ensures that the data to be exchanged exist in the participating systems as an information entity or that the necessary information can be derived from the available data, e.g., by means of aggregation or disaggregation.

- Data Transformation is the technical process - implemented often by the respective algorithms in gateways and interfaces - of aggregation and/or disaggregation of the information entities so that they match the information exchange requirements, including the adjustment of data formats. 
In reality, the majority of work has been focused on data transformation, i.e., on programming or maintenance of interfaces. However, if such efforts are not accompanied by alignment of the respective control processes for data administration, management, and alignment, the result is at best only a temporary solution, effective until the next update of one of the involved systems. Therefore, the management processes of the participating systems must at least be harmonized. In the ideal case, the program managers will even use the same methods and support tools under a common, overarching approach.

Currently, the C4ISR and the M\&S communities are in the process of establishing solutions that support these management efforts. In order to ensure continuous interoperability these processes have to be harmonized and coordinated, leading to a common approach.

\section{Data Administration}

The C4ISR community understands the process of data administration very well. For every field system a Command, Control, Communications, Computing, and Intelligence Support Plan (C4ISP) is required that describes the necessary information infrastructure needed to find the components' place in the overarching C4ISR architecture. The definition of the exact information exchange requirements is part of this plan, i.e., each information entity is defined by its syntax and semantics. The required methodologies can be found in the US DoD C4ISR Architecture Framework. ${ }^{7}$ The NATO Consultation, Command and Control (C3) System Architecture Framework is the international version of this document. ${ }^{8}$ In this respect, the $M \& S$ community has not yet reached such maturity. However, similar ideas are already part of the Common Technical Framework (CTF) included in the High Level Architecture as well as the respective Data Standards (DS) and Functional Description of the Mission Space (FDMS). ${ }^{9}$ Within these concepts, especially the idea of using a Simulation Object Model (SOM) defined by means of HLA/Object Model Template (OMT, IEEE P1516.2) comes close to the definition of a general information exchange view on the respective systems. However, in reality there is no consensus on a common approach neither for C4ISR systems nor for M\&S systems. It might not even be exaggerated if we say that the number of experts realizing the need for such a common method is still very limited.

\section{Data Management}

The process of data management is tightly related to the definition of a domain overarching ontology. The main objective is the development of a common understanding/view of the world. In this respect, the use of reference models has proven very useful. In the domain of C4ISR two models have to be mentioned 
explicitly, although this should not exclude other solutions. Within the US Army, the US Joint Common Database (JCDB) data model builds the kernel for all future Army Battle Command Systems (ABCS) versions. ${ }^{10}$ Within NATO, the Land Command and Control Information Exchange Data Model (LC2IEDM) not only provides the kernel for future C4ISR systems, but has also been used as a reference model for the NATO Data Administration Group (NDAG) whose responsibility is data management for NATO's present and future C4ISR systems. ${ }^{11}$ The main idea of data management using a reference data model is to find matching information entities in the data model being managed and the reference data model used for standardization. For each information entity in the data model under consideration, the data management agency defines a semantically equivalent standardized information entity or a semantically equivalent set of information entities including their relations. In this way, a set of standardized data elements (SDE), including respective mapping rules, is created. It is important that this process is performed following rigorous rules that extend the reference data model to insure that no redundancies or contradictions occur. It should be pointed out that the two reference data models - the JCDB and the LC2IEDM - have common roots in the NATO-hosted Permanent Working Group (APWG) of international experts in data modeling and management working over 10 years on the definition of the next generation of Allied Tactical Command and Control Information Systems (ATCCIS). The ATCCIS data model has been designed to meet exactly the required criteria for data management: the existing information exchange requirements are included and the extension rules allow redundancy and contradiction-free introduction of additional new information exchange requirements in future operations. The applicability to C4ISR and M\&S systems has been demonstrated in the United Kingdom, Germany, and the US.

\section{Data Alignment}

It is often assumed that the data describing real systems in operations - as used by C4ISR systems - and the data describing simulated systems in simulated operations as used in M\&S systems - would not differ too much. Why should the state vector of a real system differ a lot from the state vector of the simulation of that system? This has led to the implicit assumption that there exist real-world data, which can be mapped to simulated data and vice versa, thus no management process seems to be needed. However, as has been shown recently in a study prepared for the US Department of the Army, Office Director of Information Systems for Command, Control, Communications, and Computer (ODISC4), the overlap between object and data models intended for work in the same or very similar domains is surprisingly small. ${ }^{12}$ In this study, a mapping method has been developed enabling the comparison of relational data models (described using the standard IDEF1X) and object models (described by the Unified Modeling Language UML). Additionally, the study defines 
a method to measure the alignment ranging from $0 \%$ (no alignment) to $100 \%$ (total alignment). A very good overview of this methodology has been given during the first European Simulation Interoperability Workshop. ${ }^{13}$ Without going into detail, alignment greater than $50 \%$ was rarely the case. Due to the surprising results from some alignment studies conducted last year, the US Army has decided to develop for the simulation community an Army Standard C4ISR Object Model that would represent the data structures to be used in operational C4ISR systems. In parallel to the US efforts, under the aegis of the German Army Office, the data models of various German simulation systems are harmonized with the LC2IEDM in an effort to initialize a common shared data model facilitating future interoperability issues. To support this, under consideration is a requirement to conduct relevant data alignment studies as a necessary step in the procurement process of all new military information systems.

\section{Data Transformation}

Programming of interface using knowledge for data translation is the last step. In addition, a great deal of configurable gateways enters the market facilitating the process of implementing the data transformation process. However, as is pointed out in the German findings on the applicability of data federation approaches, after having agreed on a common shared data model and mapping rules for harmonization are defined and distributed by a system independent data management organization, data mediation in the sense of automatic translation of system's data into standardized data elements and vice versa becomes possible. Using an appropriate toolkit, the results of data administration, data management, and data alignment can be used directly to configure a software layer interconnecting the data access layers of different systems with heterogeneous data interpretations. It has to be pointed out that this is not an isolated technical solution to achieve interoperability between different information systems, but the result of an integrated management process and the use of common standardized tools. The applicability of this solution to coalition interoperability of C4ISR systems has been demonstrated during a recent NATO workshop on new information technologies. ${ }^{14}$ It could be concluded that the same technique, tools, and procedures can be used to ensure interoperability between C4ISR systems, as well as between C4ISR and M\&S systems. In addition, the importance of the results of this study in operational systems and the applicability of the methods to operational analyses have been the topic of a paper presented at a NATO Studies, Analyses, and Simulation Symposium. ${ }^{15}$ 


\section{A Framework for Interoperable Shared Solutions}

As already has been stated, the integration of data is only one aspect of interoperability. Figure 1 shows the "House Diagram," which illustrates the complexity of interfacing M\&S and C4ISR systems. ${ }^{16}$ This holistic view emphasizes the interdependence of the five major factors in the effort to guarantee shared solutions for C4ISR/M\&S interoperability: Architectures Alignment, Common Data/Object Models, Common Standards, Alignment Processes, and Reusable Component Interfaces.

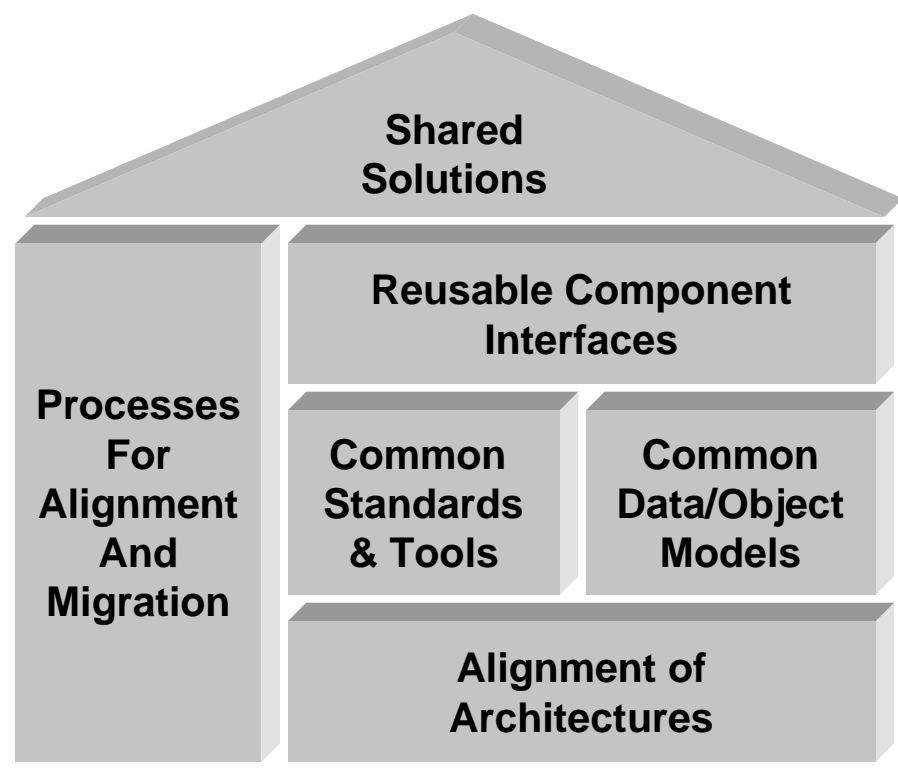

Figure 1: Components of Interoperability.

Architecture Alignment recognizes the fundamental need to align the framework architectures of the M\&S and C4ISR domains. The US DoD C4ISR community under the aegis of the Defense Information Systems Agency (DISA) has developed the Common Operating Environment. ${ }^{17}$ NATO uses the NATO Consultation, Command and Control (C3) Technical Architecture. ${ }^{18}$ The M\&S community has established the High Level Architecture. ${ }^{19}$ These efforts build the foundation for interoperability between components of these two domains. Architecture alignment has to resolve the differences in the viewpoints or in the representation of the "problem space." 
Within the M\&S domain, the HLA Federation Object Model (FOM) methodology is used to align Data Models and Object Models among M\&S federates. While this methodology works, it places a heavy burden on developers. When extended to the C4ISR domain, temporary (situational) alignment presents additional challenges: synchronizing development cycles, aligning domain ontology, and coordinating data standards. Actually, a data translation layer between C4ISR and simulation domains normally resolves these constraints. If, in the future, systems are aligned to the same or similar object or data model representations, performance increases due to the decrease of translation penalties and FOM alignment burden.

Common Standards are most effective when they are part of the system design. Integration of standards begins with the framework architecture for each domain and extends to support for common objects and data models. In other words, C4ISR and M\&S systems have to work towards interoperability from the beginning, i.e., using the same architecture framework.

Reusable Component Interfaces sit atop, and, therefore, rest on the building blocks presented below. However, compared to architectures, models, or standards, interfaces have been a hotbed of activity. This apparent paradox stems from the ability to partition the problem space at interface level and thus provide short-term solutions for quick success. However, as these solutions are in general too shallow, one has to invest again for the individual solutions in terms of costs, time, and flexibility. By realignment of the underlying structures/components basic incompatibilities between the systems can be eliminated, thus leading to a large number of benefits.

To achieve the overarching goal, these technical processes have to be accompanied by harmonization of the Management Processes for Alignment and Migration of legacy applications and systems in order to keep the parallel developments on track. This aspect is very often neglected in articles proposing technical solutions to interoperability.

Finally, the top of the diagram envisions Shared Solutions between C4ISR and Simulation systems. This objective has to be supported by all the underlying blocks. In addition, it requires that the systems align or translate the included processes. For example, terrain alignment and object placement must be consistent between the components in the two domains. These shared solutions are the objective in mid term.

\section{Future Work}

In long term, however, integrated solutions are the objective. On the C4ISR side, initial ideas have been proposed with the vision of network-centric warfare where components of M\&S and C4ISR systems work together within a common info- 
sphere. ${ }^{20}$ The application of these ideas to the domain considered in this paper is further suggested by the proposals for one command and control system based on heterogeneous information techniques. ${ }^{21}$

In addition, new developments in the sector of commercial information technologies offer very promising integration solutions, e.g., the Model Driven Architecture (MDA) developed by the Object Management Group (OMG). ${ }^{22}$ The underlying idea behind the MDA is to use a common stable model, which is language-, vendor- and middleware-neutral. This model is a meta-model of the concept. With such a model in the center of the development and integration processes, users that have adopted the MDA gain the ability to develop code for various sub-levels. And, even if the underlying infrastructure changes over time, the meta-model remains stable and can be ported to various middleware implementations, as well as, to different platforms. This approach can also combine various other concepts used nowadays to increase interoperability, e.g., the Common Object Request Broker Architecture (CORBA), Sun's Enterprise JavaBeans, Microsoft's Distributed Component Object Model (DCOM), the Extensible Markup Language (XML) and the XML Metadata Interchange (XMI) Standards, and many others (e.g., the HLA used by the M\&S community, as well as C4ISR solutions).

The time to realize these visions has come. On one hand, more military users formulate the need for operational support in an increasingly demanding military operational environment. ${ }^{23}$ There are many operational challenges that can be tackled using algorithms and ideas borrowed from the M\&S community, e.g., harmonization of operational images, aggregation and disaggregation, pattern matching, etc. On the other hand, the operational architectures being used by the C4ISR systems - the Common Operating Environment in the US and the NATO Consultation, Command and Control (C3) Technical Architecture for NATO nations - are reformulated so as to become object-oriented and open to technical solutions from other communities. The M\&S community has to be very well aware of what happens in this field in order to be able to build operationally relevant solutions.

In summary, the methodologies and the operational needs for coupling, federating, and, finally, integrating M\&S and C4ISR systems are present and ready to be applied in the near future in order to increase the overall efficiency of soldiers in military operations. 


\section{Notes}

1 DoD Modeling \& Simulation (M\&S) Master Plan, DoD Directive 5000.59-P, (Washington, D.C.: United States Department of Defense (DoD), Under Secretary of Defense for Acquisition and Technology, October 1995).

2 NATO Modelling and Simulation Master Plan, Version 1.0, AC/323 (SGMS) D/2, (Brussels, Belgium: NATO, North Atlantic Council, August 1998).

Donald H. Timian, Joseph Lacetera, Chris Wertman, Michael R. Hieb, Andreas Tolk, and Kevin Brandt, "Report Out of the C4I Study Group" (paper 00F-SIW-005 presented at the Fall Simulation Interoperability Workshop 2000, Orlando, Florida, September 2000).

A. P. Sheth and J. A. Larson, "Federated Database Systems for Managing Distributed, Heterogeneous, and Autonomous Databases," ACM Computing Surveys 22, 3 (1990): 183-236.

Stefan Conrad, Föderierte Datenbanksysteme (published in German, Translation: Federated Database Systems) (Berlin, Germany: Springer, 1997).

Andreas Tolk, "Bridging the Data Gap - Recommendations for Short, Medium, and Long Term Solutions" (paper 01S-SIW-011 presented at the Spring Simulation Interoperability Workshop 2001, Orlando, Florida, March 2001).

C4ISR Architecture Framework Version 2.0 (Washington, D.C.: US DoD C4ISR Architecture Working Group (AWG), December 1997).

NATO C3 System Architecture Framework, AC/322-WP/0125, (Brussels, Belgium: NATO Consultation, Command and Control Board (NC3B), November 2000).

Paul Murtha and Jim Campbell, "Basics of Modeling and Simulation in DoD" (Tutorial (MS 101) presented at the Interservice/Industry, Simulation and Education Conference (I/ITSEC), Orlando, Florida, November 2001).

Michael R. Hieb and James Blalock, "Data Alignment between Army C4I Databases and Army Simulations" (paper 99S-SIW-034 presented at the Spring Simulation Interoperability Workshop 1999, Orlando, Florida, March 1999).

11 The Land C2 Information Exchange Data Model, Edition 2.0, Allied Data Publication (ADat-P) 32, (Brussels, Belgium: NATO, March 2000).

12 Steven Wartik, Brian Haugh, Michael Hieb, and Francisco Loaiza, "Alignment of Army Integrated Core Data Model and Object Management Standards Category," Paper P3596, (Alexandria, Virginia: Institute for Defence Analyses, February 2002).

13 Steven Wartik, Brian Haugh, Francisco Loaiza, and Michael Hieb, "A Methodology for Comparing C4I Data Models and Simulation Object Models" (paper 01E-SIW-059 presented at the 2001 European Simulation Interoperability Workshop, London, United Kingdom, June 2001).

14 Stefan Krusche and Andreas Tolk, "Information Processing as a Key Factor for Modern Federations of Combat Information Systems" (paper presented at the NATO IST Panel Symposium on New Information Processing Techniques for Military Systems, RTO-MP049, Istanbul, Turkey, October 2000).

15 Mark R. Sinclair and Andreas Tolk, "Building up a Common Data Infrastructure" (paper presented at the NATO Studies, Analysis and Simulation (SAS) Symposium on "Analyses of the Military Effectiveness of Future C2 Concepts and Systems," The Hague, The Netherlands, April 2002). 
Michael R. Hieb et al., "Interim Report of the C4ISR/Simulation Technical Reference Model Study Group" (paper 01F-SIW-094 presented at the Fall Simulation Interoperability Workshop 2001, Orlando, Florida, September 2001).

The Common Operating Environment (US DoD, Defense Information Systems Agency (DISA)), <http://diicoe.disa.mil/coe/> (July 2002).

NATO C3 Technical Architecture, Allied Data Publication (ADat-P) 34, AC/322(SC/5)WP/31, (Brussels, Belgium: NATO Open Systems Working Group, October 2000).

IEEE Standard 1516, High Level Architecture, IEEE P1516: Framework and Rules; IEEE P1516.1: Federate Interface Specification; IEEE P1516.2: Object Model Template (OMT) Specification.

G. D. Peterson and J. D. Birdwell, "Designing and Validating the Joint Battlespace Infosphere," in Proceedings of SPIE 4396, (Orlando, Florida, April 2001).

Andreas Tolk, "Heterogeneous Synergy - A Vision for the Next Generation of Warfighter IT systems" (paper 00F-SIW-013 presented at the Fall Simulation Interoperability Workshop 2000, Orlando, Florida, September 2000).

Richard Soley and the OMG Staff Strategy Group, "Model Driven Architecture," White Paper (Object Management Group (OMG), November 2000) < www.omg.org/ $\sim$ soley/mda.html> (July 2002).

Andreas Tolk, "Functional Categories of Support to Operations in Military Information Systems" (paper presented at (a) NATO Regional Conference on Military Communication and Information Systems 2000, Zegrze, Poland, October 2000, and (b) Army Operations Research Symposium XXXIX (39. AORS), Fort Lee, Virginia, October 2000).

ANDREAS TOLK is a Senior Research Scientist at the Virginia Modeling Analysis and Simulation Center (VMASC) in Suffolk, which is a not-for-profit collaborative enterprise center at the Old Dominion University's College of Engineering and Technology in Norfolk, Virginia. As a chairman of the C4I Forum of the Simulation Interoperability Standards Organization (SISO), he has been actively engaged in interoperability issues of M\&S and C4I systems for several years. He received his Ph.D. in Computer Science/Military Operations Research from the University of the Federal Armed Forces of Germany, Munich, Germany, in 1995 for his work on integrating Means of Artificial Intelligence into Closed Combat Simulation Systems. He has published over 30 papers and articles in the domain of military applications of information technologies, focusing on M\&S and C4ISR. Before joining VMASC, he had worked in Germany for the main contractors of the German DoD for Operations Research (IABG, Munich, Germany) as well as for C4ISR systems (ESG, Munich, Germany). He has also participated in several NATO activities in these domains. He holds a Master of Science degree in Computer Science and teaches for the M\&S program at the Old Dominion University and in the Lecture Series for NATO. Email: atolk@odu.edu. 